\title{
Constraining the dark energy dynamics with the cosmic microwave background bispectrum
}

\author{
Fabio Giovi ${ }^{1}$, Carlo Baccigalupi ${ }^{1,2}$, Francesca Perrotta ${ }^{1,2}$ \\ ${ }^{1}$ SISSA/ISAS, Via Beirut 4, 34014 Trieste, Italy \\ ${ }^{2}$ LBNL, 1 Cyclotron Road, 94720 Berkeley, CA, USA
}

\begin{abstract}
We consider the influence of the dark energy dynamics at the onset of cosmic acceleration on the Cosmic Microwave Background (CMB) bispectrum, through the weak lensing effect induced by structure formation. We study the line of sight behavior of the contribution to the bispectrum signal at a given angular multipole $l$ : we show that it is non-zero in a narrow interval centered at a redshift $z$ satisfying the relation $l / r(z) \simeq k_{N L}(z)$, where the wavenumber corresponds to the scale entering the non-linear phase, and $r$ is the cosmological comoving distance. The relevant redshift interval is in the range $0.1 \lesssim z \lesssim 2$ for multipoles $1000 \gtrsim l \gtrsim 100$; the signal amplitude, reflecting the perturbation dynamics, is a function of the cosmological expansion rate at those epochs, probing the dark energy equation of state redshift dependence independently on its present value.

We provide a worked example by considering tracking inverse power law and SUGRA Quintessence scenarios, having sensibly different redshift dynamics and respecting all the present observational constraints. For scenarios having the same present equation of state, we find that the effect described above induces a projection feature which makes the bispectra shifted by several tens of multipoles, about 10 times more than the corresponding effect on the ordinary CMB angular power spectrum.
\end{abstract}

\section{INTRODUCTION}

The recent cosmological observations indicate that the Universe is nearly geometrically flat, filled with structures which grew out of a primordial linear spectrum of nearly Gaussian and scale invariant perturbations; the scalar contribution, i.e. from the perturbations in the energy density, appears to dominate over the tensor one from cosmological gravitational waves. About $5 \%$ of the critical energy density is made of baryons, while the remaining dark part is supposed to interact at most weakly with the baryons themselves, since we observe it only indirectly, i.e. through its gravitational effects. The dark component appears to be $30 \%$ pressureless, like in Cold Dark Matter (CDM) scenarios, dominating the gravitational potentials perturbations which host visible structures like galaxies or clusters. The remaining $70 \%$ should be in some sort of vacuum energy, with negative pressure acting as a repulsive gravity, and responsible for a late time cosmic acceleration era.

Despite of the remarkable convergence of the most important cosmological parameters, the picture is clearly far from being satisfactory: without a better insight into the nature of the dark cosmological component, we cannot claim to have a satisfactory physical understanding of cosmology. In particular, the evidence for a non-zero vacuum energy driving cosmic acceleration today (hereafter dark energy) is a clear sign that the study of the dark cosmological component could have unexpected and most important consequences on cosmology and more generally on fundamental physics. The evidence for the dark energy is rather robust, since it comes from two independent datasets. The first one is represented by the observations of distant standard candles, namely type Ia supernovae (hereafter SNIa), directly probing the cosmological expansion rate at low redshifts [1]; these observations indicate that a $70 \%$ fraction of the critical energy density today is in the dark energy. The same result is supported, independently, by the observations of the Cosmic Microwave Background (CMB) anisotropies and Large Scale Structure (LSS). The abundance of clustered matter is known to be at the $30 \%$ level of the critical density by observing the nearby Universe [2]; on the other hand, the scale subtended in the sky by the acoustic oscillations in the CMB anisotropy angular power spectrum clearly support a flat cosmological geometry (see the recent results by the Wilkinson Microwave Anisotropy Probe, map.gsfc.nasa.gov, hereafter WMAP, [3] and references therein). Thus, CMB and LSS together indicate again a $70 \%$ of critical energy density in the vacuum. The only way to break this evidence would be invoking a low value of the Hubble constant, which is excluded by the Hubble Space Telescope (HST) data [4].

In cosmology, a constant vacuum energy density is provided by the Cosmological Constant. While a dark matter component has well established support from the theories beyond the standard model of particle physics, a Cosmological Constant at the observed level raises two fundamental questions. The first is the fine-tuning: the energy scale is about 120 orders of magnitude less of the Planck energy density which is supposed to be the unification scale of all forces in the early Universe. The second question is the coincidence: not only the vacuum energy scale is small with respect to any fundamental scale, but it is such that it is comparable to the matter energy density today.

Already several years ago [5, 6] it was realized that the evidence for a Cosmological Constant would have raised these crucial questions. To address them, the Cosmological Constant was minimally extended to a dynamical and inhomogeneous scalar field, evolving slowly enough to yield an almost constant vacuum energy out of its potential, 
providing cosmic acceleration. The latter evidence caused a renewed interest in these models [7, 8]. In particular, it was demonstrated how the dynamics of this component, under suitable potential energy, can originate attractors in the trajectory space, named tracking solutions, capable to reach the present dark energy density starting for a in the remote very early Universe, thus alleviating, at least classically, the problem of fine-tuning mentioned above [9, 10]. The scalar field playing the role of the dark energy was named Quintessence. This description allowed to investigate the relation of the dark energy with the other cosmological components: while an explicit coupling with baryons is severely constrained by observations [1] , the relation of the Quintessence with the gravitational 12] and dark matter 13. sectors of the fundamental Lagrangian, as well as the phenomenology arising from generalized kinetic energy terms 14 have been extensively studied. The Quintessence field has been proposed to be related to super-symmetry and super-gravity theories (see [15] and [16], respectively, and references therein) but we still lack a reliable candidate from fundamental physics.

Generally speaking, despite of the large number and variety of ideas proposed to explain the evidence for cosmic acceleration, going from quantum spacetime microphysics to exotic particle physics (see [17] and references therein), no valid solution to the coincidence problem has been provided yet. It is likely that a breakthrough in the understanding of the dark energy could come from the future high precision data on the redshift dependence cosmological acceleration. Indeed, almost all the candidates for the dark energy predict a characteristic behavior of the equation of state, $w$, as a function of the redshift $z$ (see [18 and references therein). A good example of this occurrence, which we will use extensively in this work, are the different Quintessence potentials proposed so far, causing relevant differences in $w(z)$ at redshift relevant for cosmic acceleration, even if its present value is close to the Cosmological Constant case, $w_{0}=-1[19]$.

The recent CMB observations, combined with the LSS, HST and SNIa data, allow to constrain the effective dark energy equation of state, assumed to be constant in time, to be $w_{\text {eff }}<-0.78$ at $2 \sigma$ confidence level imposing $w>-1$ prior and $w_{\text {eff }}=-0.98 \pm 0.12$ dropping the previous prior (see [20] and references therein). That will be certainly improved by future observations, but the real challenge in order to discriminate between different models is to gain insight into the dark energy equation of state redshift dependence. Together with the observations of distant SNIa, it is likely that the study of cosmological structure formation will be crucial for investigating the onset of cosmic acceleration, since the redshift intervals relevant for the two processes overlap. The best way to look at the structure formation process is just to study the weak gravitational lensing deflection induced by forming structures along the line of sight of the background light (see 21] for reviews). The shear induced by weak lensing on background galaxies has been observed by several groups, and the results agree impressively between different telescopes and data reduction techniques [22]; already, several dark energy weak lensing observables have been proposed [23]. New powerful probes to map the weak lensing shear over large sky areas are being designed to operate in the next decade with ground-based and space surveys (see 24] and references therein). On the CMB side, the weak lensing effect on the total intensity and polarization CMB anisotropies can be predicted on the general basis of cosmological perturbation theory [25]. The effect is generally non-Gaussian, and relevant at the arcminutes angular scale; it is likely that the low noise, arcminute resolution imaging expected by the Planck satellite (see astro.estec.esa.nl/SA-general/Projects/Planck/) and future CMB missions (see CMBpol spacescience.nasa.gov/missions/concepts.htm) will have a crucial importance in weak lensing studies.

The weak lensing effect on the CMB is the subject of the present work. We focus on the third order statistics, where the background CMB light, if well described by a Gaussian process at last scattering as it appears from the recent CMB observations [26], yields a zero contribution within cosmic variance. As we already mentioned, a suitable harmonic approach to the weak lensing effects on the CMB total intensity and polarization has been carried out [25], out of a general description of the CMB anisotropies [27]. Further works focused specifically on the effects on the CMB bispectrum [28], and in particular on the benefits of having bispectrum data in addition to the CMB power spectrum, allowing more precise estimates of the main cosmological parameters, even breaking the degeneracies when the dark energy is included into the analysis [29]. In this work we investigate the sensitivity of the bispectrum to the redshift dependence of the dark energy equation of state $w(z)$.

In Section $\amalg$ we review the basic features of the Quintessence dynamics in tracking trajectories, which we will use later to illustrate the phenomenology we study. In Section [II] we review the CMB third order statistics, described through the bispectrum, and how it is affected through the weak gravitational lensing induced by structure formation. In Section [IV we study the bispectrum line of sight integral describing how it is sensitive to the cosmological expansion rate redshift dependence, and in Section $\nabla$ we provide a first quantification of the effect by considering the leading Quintessence scenarios. Finally, in Section VI we make the concluding remarks. 


\section{QUINTESSENCE COSMOLOGY}

The dynamics of the Quintessence scalar field $\phi$ in cosmology follows the Klein-Gordon equation, describing background and linear perturbation evolution:

$$
\square \phi+V^{\prime}=0, \delta(\square \phi)+V^{\prime \prime} \delta \phi=0,
$$

where the $\square$ is the Dalambertian operator in a Friedmann Robertson Walker (FRW) cosmology, and $\delta$ represents linear fluctuation, while $V(\phi)$ is the Quintessence potential and $V^{\prime}=d V / d \phi$.

We consider here inverse power law (hereafter RP, first considered in [5]) and SUGRA [16] potentials, defined respectively as

$$
V_{R P}=\frac{M^{4+\alpha}}{\phi^{\alpha}}, V_{S U G R A}=\frac{M^{4+\alpha}}{\phi^{\alpha}} \cdot \exp \left(4 \pi G \phi^{2}\right) .
$$

The exponential in the SUGRA model induces relevant changes in the field dynamics with respect to the RP case, as we explain now. As we mentioned in the Introduction, the tracking trajectories in the Quintessence scenarios alleviate the fine-tuning in the early Universe, since by means of them one is no longer forced to assume that the dark energy is vanishingly small with respect to radiation in the early Universe [5, 6, 8, 9, 10]: the present field value $\phi_{0}$, of the order of the Planck mass $M_{\text {Planck }}$, is reached from a wide set of initial conditions $\phi_{i}$ and $\dot{\phi}_{i}$, with the only important condition that $\phi_{i} \ll M_{\text {Planck. }}$. Until the latter regime is satisfied both in the RP as well as the SUGRA cases, the tracking trajectories are well defined until the Quintessence is subdominating, yielding an almost constant equation of state obeying the simple relation

$$
w=\frac{p_{D E}}{\rho_{D E}}=-\frac{2}{2+\alpha},
$$

where $p_{D E}=(d \phi / d t)^{2} / 2-V$ and $\rho_{D E}=p_{D E}+2 V$ are the field pressure and energy density, respectively. In the $\mathrm{RP}$ scenario, if we require the present equation of state to be in agreement with the current constraints [3] , say $-1 \lesssim w_{\text {eff }} \lesssim-0.8$, the exponent is in the range $0 \lesssim \alpha \lesssim 0.5$, yielding a shallow potential shape. At the present, the tracking regime is abandoned because the dark energy is no longer a subdominant component, but the shallow potential shape makes the present equation of state not far from the tracking one in Eq. (3), differing typically at the $10 \%$ level. The SUGRA exponential correction flattens sensibly the potential shape at $\phi \simeq M_{\text {Planck }}$, i.e. at the end of the tracking trajectory. This means that a given equation of state at present is obtained for values of $\alpha$ sensibly larger than in the RP case, meaning that the dark energy dynamics and thus the cosmological expansion rate as a function of the redshift are generally much different in the two cases. This aspect is phenomenologically illustrated in Fig. 1] featuring the redshift evolution of the equation of state in SUGRA and RP tracking trajectories yielding at present $w_{0}=-0.9$, from the present to the tracking regime; we consider a cosmological scenario which is close to the current best fit [3], i.e. a flat Friedmann Robertson Walker (FRW) cosmology with Hubble parameter $h=0.7$, baryon, CDM and dark energy fraction density $\Omega_{b} h^{2}=0.022, \Omega_{C D M}+\Omega_{b}=0.3, \Omega_{D E}=0.7$, respectively, three species of massless neutrinos and a scale invariant scalar perturbation spectrum with no gravitational waves. The behavior of $w(z)$ in the figure is markely different in the two Quintessence scenarios considered, reflecting the two values of $\alpha$ required to have $w_{0}$; specifically, $\alpha=0.34$ and 1.76 in the RP and SUGRA cases, respectively. We will also consider an effective dark energy model with constant $w=-0.9$ (hereafter $\mathrm{DE}_{\text {eff }}$ ) obtained simply by giving that equation of state to the Cosmological Constant term in the FRW background cosmological evolution. That is physically incorrect because it assumes no spatial fluctuations in the dark energy; the latter are present indeed, and play a relevant role even in the minimally coupled Quintessence scenarios on super-horizon scales, as it can be seen by looking at the large scale CMB anisotropies [30]; in addition, the dark energy spatial fluctuations are present on all scales if the field interacts with other cosmological components [1, 12, 13]. The curves in Fig. 1] as well as all the perturbation spectra quantities in the next Section, have been numerically computed by making use of a dark energy oriented version [31] of the CMBFAST code [32].

The most relevant quantities for the following discussion are the distance and the dark energy density behavior as a function of the redshift $z$. The first is given by

$$
r(z)=\frac{c}{H_{0}} \int_{0}^{z} \frac{d z^{\prime}}{\sqrt{\Omega_{M 0}\left(1+z^{\prime}\right)^{3}+\rho_{D E}\left(z^{\prime}\right) / \rho_{c 0}}},
$$

where we neglected the radiation contribution and $\rho_{c 0}$ is the critical cosmological density at present; $\rho_{D E}(z)$ is the redshift dependent background dark energy density, given by

$$
\rho_{D E}(z)=\rho_{D E 0} \exp \left[3 \int_{0}^{z} d z^{\prime} \frac{1+w\left(z^{\prime}\right)}{1+z^{\prime}}\right],
$$




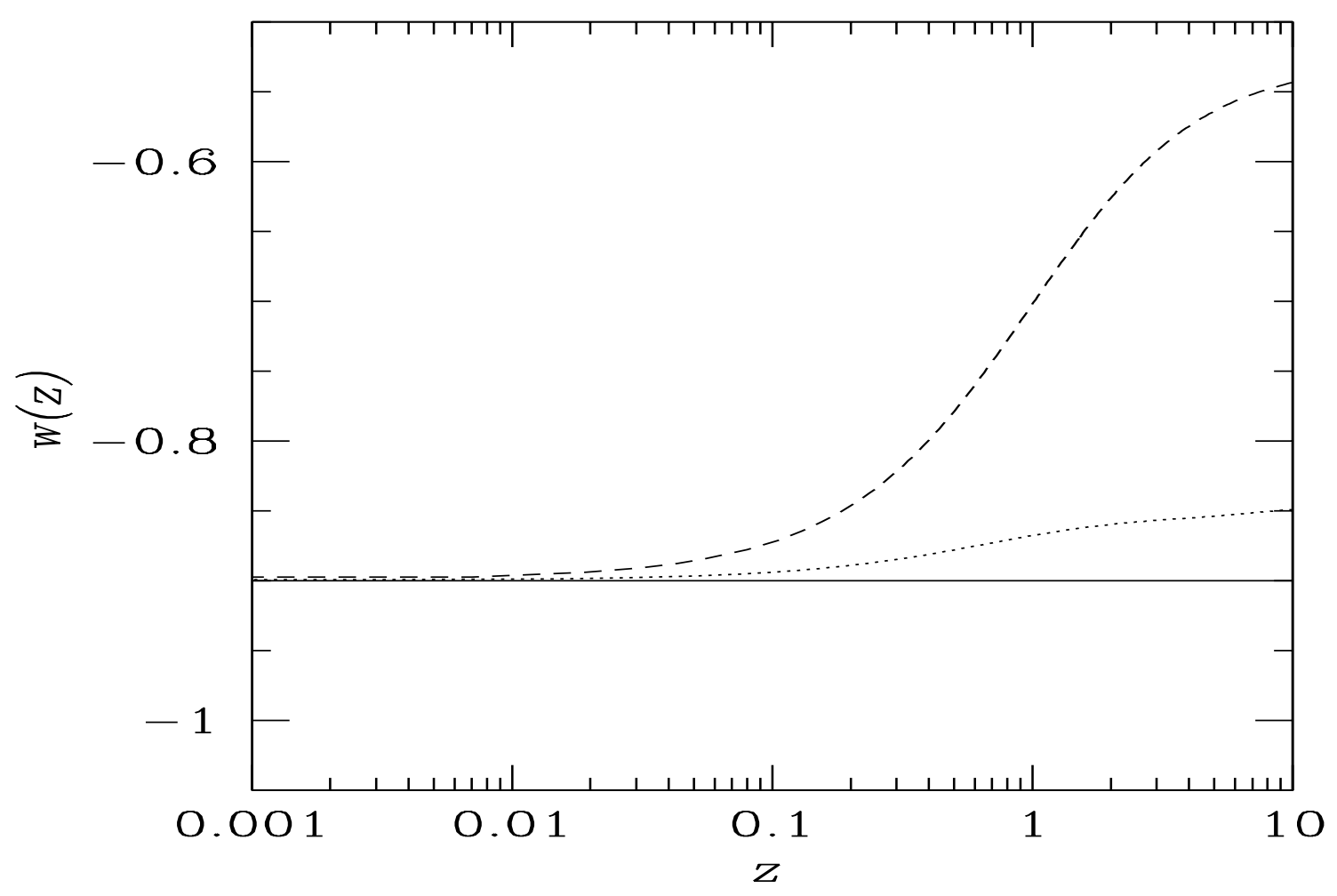

FIG. 1: Equation of state redshift dependence for the Quintessence models described in the text, for the RP model (dotted line) and the SUGRA (dashed line). Note that both have $w_{0}$ at the present. The solid line is a DE $E_{\text {eff }}$ model with a constant equation of state.

as it can be verified by simply solving the energy conservation equation. In the RP and SUGRA scenarios described above, $r(z)$ and $\rho_{D E}(z)$ have different behavior as a consequence of the different behavior of $w(z)$ shown in Fig. 1

We conclude this Section by recalling briefly the main dark energy effects of the CMB angular power spectrum 33$]$. As $w$ gets larger than -1, the distance in Eq. (4) gets reduced. This causes a shift of the CMB angular power spectrum toward smaller multipoles, i.e. large angular scales, affecting in particular the acoustic peaks location. Moreover, for $w>-1$ the dark energy domination era begins earlier that in for the Cosmological Constant, since $\rho_{D E}$ increases with redshifts. This boosts the gravitational potential dynamics at low redshift, injecting dynamics on large angular scales, where however the Cosmic Variance greatly limits the impact of the effect. Thus the projection represents the main dark energy effect on the CMB angular power spectrum. However, it should be noted that a limiting factor for the CMB power spectrum as a probe of the dark energy is the fact that the CMB path performs a sort of redshift average of the dark energy properties along their line of sight, making the CMB angular power spectrum mainly sensitive to the redshift average of the dark energy equation of state in the interval in which it is relevant, say $z \lesssim 2$, quite low with respect to the origin of the $\mathrm{CMB}$ at $z \simeq 1000$; the latter statement could be violated if the dark energy is not subdominant at last scattering [33. Nevertheless, it should be noted that a great improvement in that respect is expected by the next generation CMB satellites (see [34] for the Planck case).

On the other hand, the injection of power in the CMB anisotropies through the forming structures is potentially a stronger dark energy probe, since the relevant redshift interval is close to the onset of cosmic acceleration, as we pointed out already. This process is likely to alter the statistical properties of the CMB last scattered radiation [25]. In particular, if the latter is Gaussian as it appears from the recent CMB observations [26], it is convenient to design CMB observables looking where the noise due to the last scattered signal is lower, for example in the third order statistics. In the following we focus on this aspect. 


\section{WEAK LENSING AND CMB BISPECTRUM}

In this Section we write down the equations describing the weak lensing effect induced by structure formation on the CMB bispectrum. We decompose the CMB anisotropy in a direction $\hat{n}$ into two terms:

$$
\Theta(\hat{n}) \simeq \Theta_{l s s}(\hat{n}+\vec{\alpha})+\Theta_{I S W}(\hat{n})
$$

the first term represents the CMB anisotropy at decoupling (lss means last scattering surface), which was last scattered on a direction $\hat{n}+\vec{\alpha}$ and gravitationally lensed to our line of sight $\hat{n}$; the second term is the CMB anisotropy contribution from the forming structures along the line of sight, the Integrated Sachs-Wolfe effect (hereafter ISW [27], also known as Rees-Sciama [35]). We do not consider here the contributions coming from the Sunyaev-Zel'dovich effect [36], which yields different power at different frequencies, and the Ostriker-Vishniac effect [37]. To the first order in $\vec{\alpha}$, Eq. (6) becomes

$$
\Theta(\hat{n}) \simeq \Theta_{l s s}(\hat{n})+\Theta_{I S W}(\hat{n})+\vec{\nabla} \Theta_{l s s}(\hat{n}) \cdot \vec{\alpha},
$$

and the ISW term can be expressed as

$$
\Theta_{I S W}(\hat{n})=2 \int d r \frac{\partial}{\partial \eta} \Psi(\eta, \hat{n} r)
$$

where $\eta$ is the cosmological conformal time, $r$ the comoving distance along the line of sight, and $\Psi$ is the cosmological gravitational potential, defined as the fluctuation in the metric $d s^{2}=a^{2}\left[\left(1+\frac{2 \Psi}{c^{2}}\right) d t^{2}-\left(1-\frac{2 \Psi}{c^{2}}\right) d x^{2}\right]$, if the anisotropic stress is zero [25, 27].

Note that in general Eq. (8) describes the contribution from linear and non-linear density fluctuations, with the only assumption that the gravitational potential fluctuations they induce are linear. The effect of gravitational lensing on the CMB is contained into the last term of Eq. (7), indeed, $\vec{\alpha}$ is the gradient of the lensing potential defined as the projection of gravitational potential along the line of sight (see [21, 38]):

$$
\phi(\hat{n})=-2 \int_{0}^{r_{l s s}} d r \frac{r_{l s s}-r}{r_{l s s} r} \Psi(r, \hat{n} r) .
$$

Note that the ISW and lensing effects are correlated since they both arise from $\Psi$; this correlation induces a nonGaussian feature on the CMB pattern and leads to a non vanishing bispectrum signal [25, 28, 29, 39].

The gravitational potential $\Psi$ is built out of the density fluctuations through the Poisson equation. To describe the non-linear part of the power spectrum, we adopt a semi-analytic prescription [40] which evaluate the non-linear power out of a remapping and rescaling of the linear one, as we describe below; note that, as we show explicitly in the next Section, our results do not depend on the particular recipe we adopted for treating the non-linear density perturbations, which have been described in several ways (see for example [41] and references therein), but mainly on the perturbation dynamics in linear regime. The non-linear $(N L)$ and linear $(L)$ density fluctuation power spectra are related as

$$
P_{N L}(k, z)=G\left[\frac{4 \pi k^{3} P_{L}(k, z)}{f^{3 / 2} \sigma_{8}(z)^{\beta}}, z\right]\left(\frac{k_{L}}{k}\right)^{3} P_{L}\left(k_{L}, z\right),
$$

where the linear one is evaluated at the wavelength

$$
k_{L}=\frac{k}{\left[1+4 \pi k^{3} P_{N L}(k, z)\right]^{1 / 3}} ;
$$

the linear power spectrum is defined as usual as

$$
P_{L}(k, z)=A k^{n} T^{2}(k, z),
$$

where $A$ is the primordial perturbation amplitude, $n$ is the spectral index, and $T(k, z)$ is the matter transfer function. Note that Eq. (10) with (11) represents an implicit equation which has to be solved for $P_{N L}(k, z)$ for any wavenumber $k$ at any epoch $z$. In the relation (10) $G$ is a function describing the rise of the power spectrum at the onset of non-linearity; specifically, a suitable fit is $G(x, z)=$ $[1+\ln (1+x / 2)]\left[1+0.02 x^{4}+1.08 \cdot 10^{-4} x^{8} / g(z)\right] /\left(1+2.1 \cdot 10^{-5} x^{15 / 2}\right)$, with $\beta=0.83$ and $f=g_{0}\left|w_{0}\right|^{1.3\left|w_{0}\right|-0.76}$ 


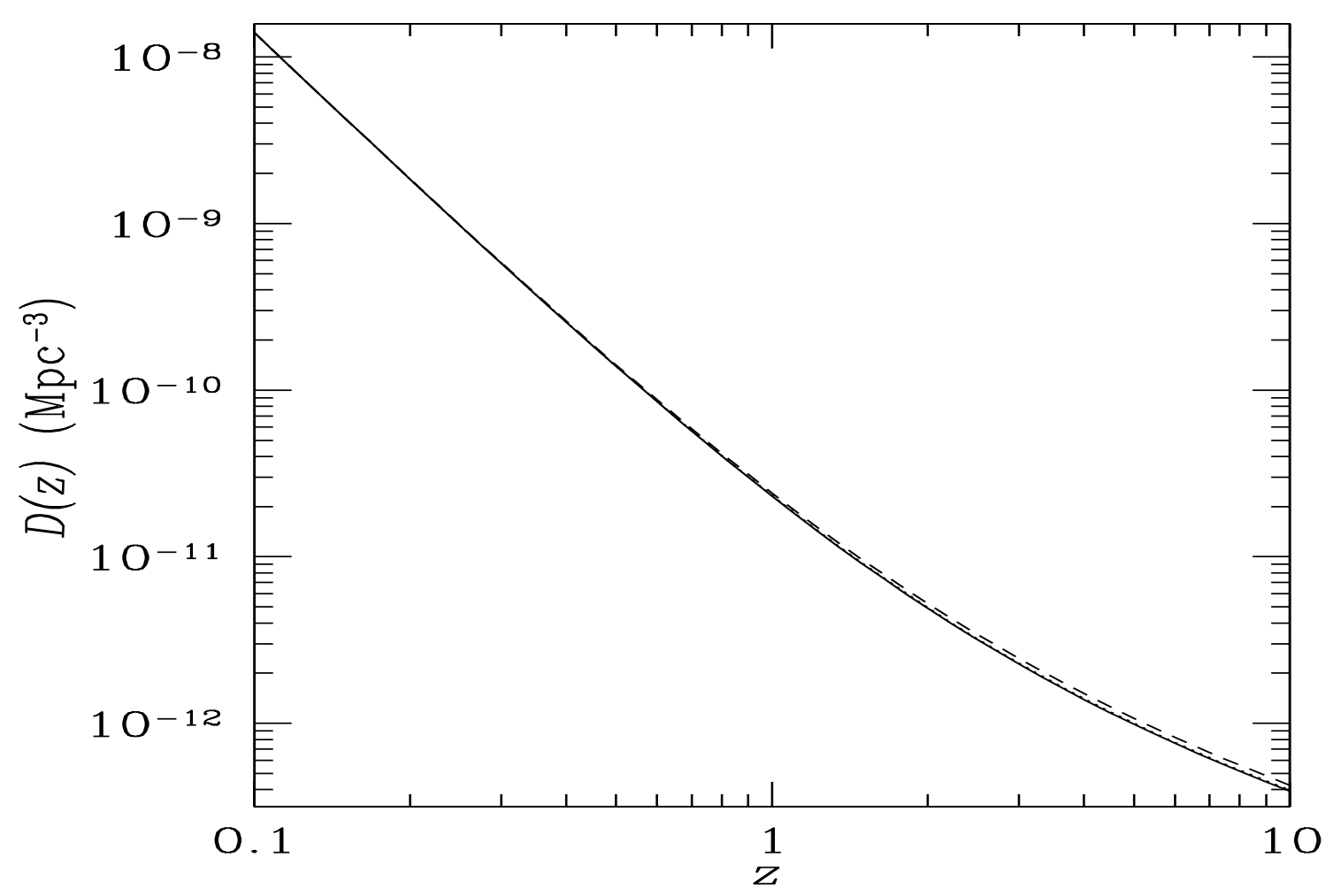

FIG. 2: $D$ factor as function of redshift for our three models: solid line is $\mathrm{DE}_{e f f}$, dotted line is RP and dashed line is SUGRA. The three curves, almost superposed, do not show significant differences between the three models.

[40]. We are interested in the case in which the dark energy equation of state changes with time. The latter modifies the background dynamics and therefore the perturbation growth rate, defined as usual as

$$
g(z)=g_{0}(1+z) \frac{T(k \rightarrow 0, z)}{T(k \rightarrow 0,0)} .
$$

Thus $w(z)$ enters in the linear perturbation growth rate as well as in the normalization $\sigma_{8}$ as a function of the redshift. As we mentioned in the previous Section, all the quantities above, i.e. $A, T(k, z)$ and $g(z)$, as well as $\sigma_{8}(z)$ are computed numerically in the Quintessence cosmologies of interest here [31]; the perturbation amplitude $A$ is determined normalizing the corresponding CMB power spectrum to the WMAP data.

The CMB bispectrum is built out of the third order statistics in the harmonic domain, and it is defined as

$$
B_{l_{1} l_{2} l_{3}}^{m_{1} m_{2} m_{3}}=a_{l_{1} m_{1}} a_{l_{2} m_{2}} a_{l_{3} m_{3}},
$$

where $a_{l m}$ are the coefficients of the expansion of the total intensity CMB fluctuations into spherical harmonics; we choose to work with dimensionless quantities when treating the CMB: in particular the $a_{l m}$ are dimensionless corresponding to the harmonic expansion of $\Delta T / T_{0}$ where $T_{0}$ is the present CMB thermodynamical temperature. If the statistics of the signal is rotationally invariant, it is convenient to work with the so called angle-averaged bispectrum [28]:

$$
B_{l_{1} l_{2} l_{3}}=\sum_{m_{1} m_{2} m_{3}}\left(\begin{array}{ccc}
l_{1} & l_{2} & l_{3} \\
m_{1} & m_{2} & m_{3}
\end{array}\right) B_{l_{1} l_{2} l_{3}}^{m_{1} m_{2} m_{3}}
$$

where the parenthesis are the Wigner 3J symbols. Expanding in spherical harmonics Eq. (77), the expansion coefficients [28, 39] can be written as

$$
\begin{aligned}
a_{l m}= & a_{l m}^{l s s}+a_{l m}^{I S W}+\sum_{l^{\prime} l^{\prime \prime}, m^{\prime} m^{\prime \prime}}(-1)^{m+m^{\prime}+m^{\prime \prime}} G_{-m+m^{\prime}+m^{\prime \prime}}^{l+l^{\prime}+l^{\prime \prime}} \\
& \frac{l^{\prime}\left(l^{\prime}+1\right)-l(l+1)+l^{\prime \prime}\left(l^{\prime \prime}+1\right)}{2}\left(a_{l^{\prime} m^{\prime}}^{l s s}\right)^{*}\left(a_{l^{\prime \prime}-m^{\prime \prime}}^{l e n s}\right)^{*},
\end{aligned}
$$



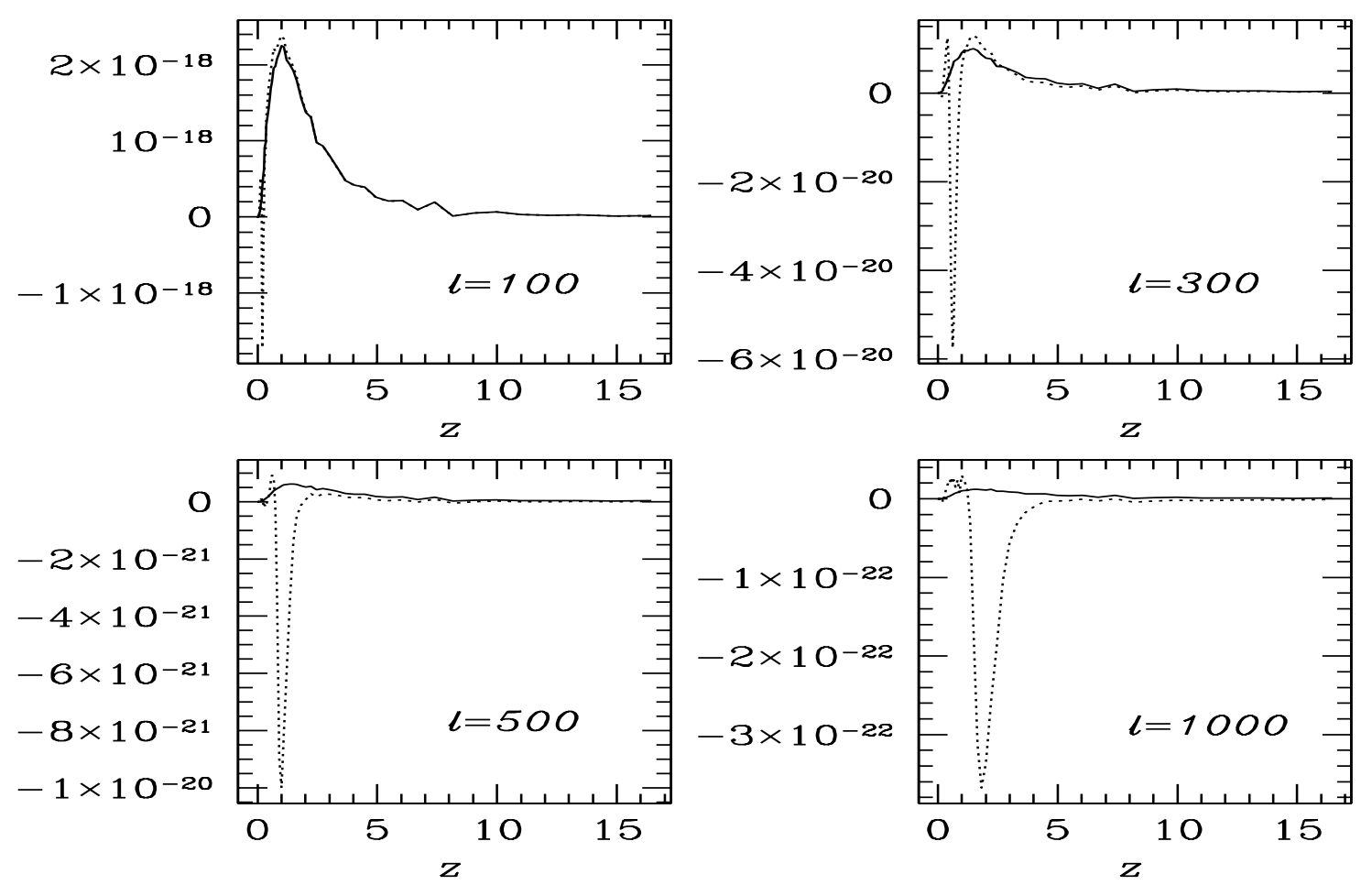

FIG. 3: Integrand for SUGRA model with $w_{0}=-0.9$ as function of redshift for different multipoles; solid line is linear regime, dotted line is non linear regime. The graphics are qualitative the same for $\mathrm{DE}_{\text {eff }}$ and $\mathrm{RP}$, the integrand is dimensionless.

where $a_{l m}^{\text {lens }}$ are the coefficients of the harmonic expansion of Eq. (9); in the equation above $G_{m_{1} m_{2} m_{3}}^{l_{1} l_{2} l_{3}}$ is the Gaunt integral defined in appendix $\mathrm{A}$ After some algebra (see the appendix in 29] for a detailed derivation), the bispectrum can be expressed by exploiting the expression above as

$$
\begin{aligned}
& B_{l_{1} l_{2} l_{3}}=\sqrt{\frac{\left(2 l_{1}+1\right)\left(2 l_{2}+1\right)\left(2 l_{3}+1\right)}{4 \pi}}\left(\begin{array}{ccc}
l_{1} & l_{2} & l_{3} \\
0 & 0 & 0
\end{array}\right) . \\
& \frac{l_{1}\left(l_{1}+1\right)-l_{2}\left(l_{2}+1\right)+l_{3}\left(l_{3}+1\right)}{2} C_{l_{1}}^{l s s} Q\left(l_{3}\right)+5 P .
\end{aligned}
$$

where $5 P$. indicates the permutation over $l_{1}, l_{2}$ and $l_{3}, C_{l}^{l s s}$ is the power spectrum of primordial CMB anisotropies, and

$$
Q(l) \equiv\left\langle\left(a_{l m}^{l e n s}\right)^{*} a_{l m}^{I S W}\right\rangle \simeq 2 \int_{0}^{z_{l s s}} d z \frac{r\left(z_{l s s}\right)-r(z)}{r\left(z_{l s s}\right) r^{3}(z)}\left[\frac{\partial P_{\Psi}(k, z)}{\partial z}\right]_{k=\frac{l}{r(z)}}
$$

where $P_{\Psi}(k, z)$ is the gravitational potential power spectrum, related to the density power spectrum by the relation $P_{\Psi}(k, z)=\left(\frac{3}{2} \Omega_{M 0}\right)^{2}\left(\frac{H_{0}}{c k}\right)^{4} P(k, z)(1+z)^{2}$, and $z_{l s s}$ is the redshift of last scattering surface; $Q(l)$ is the most relevant quantity here, describing how the forming structures along the line of sight induce the lensing on CMB photons, expressed as the statistical expectation of the correlation between the ISW and lensing effects 29, 39, 42]. In particular, the expression above has been used to evaluate the bispectrum dependence on the most important cosmological parameters, including an effectively constant dark energy equation of state, and the benefits of the bispectrum data on the estimation of the cosmological parameters themselves [29]. In the next Section we study how the contribution to $Q(l)$ is distributed along the line of sight, and how it depends on the dark energy equation of state redshift dependence. 


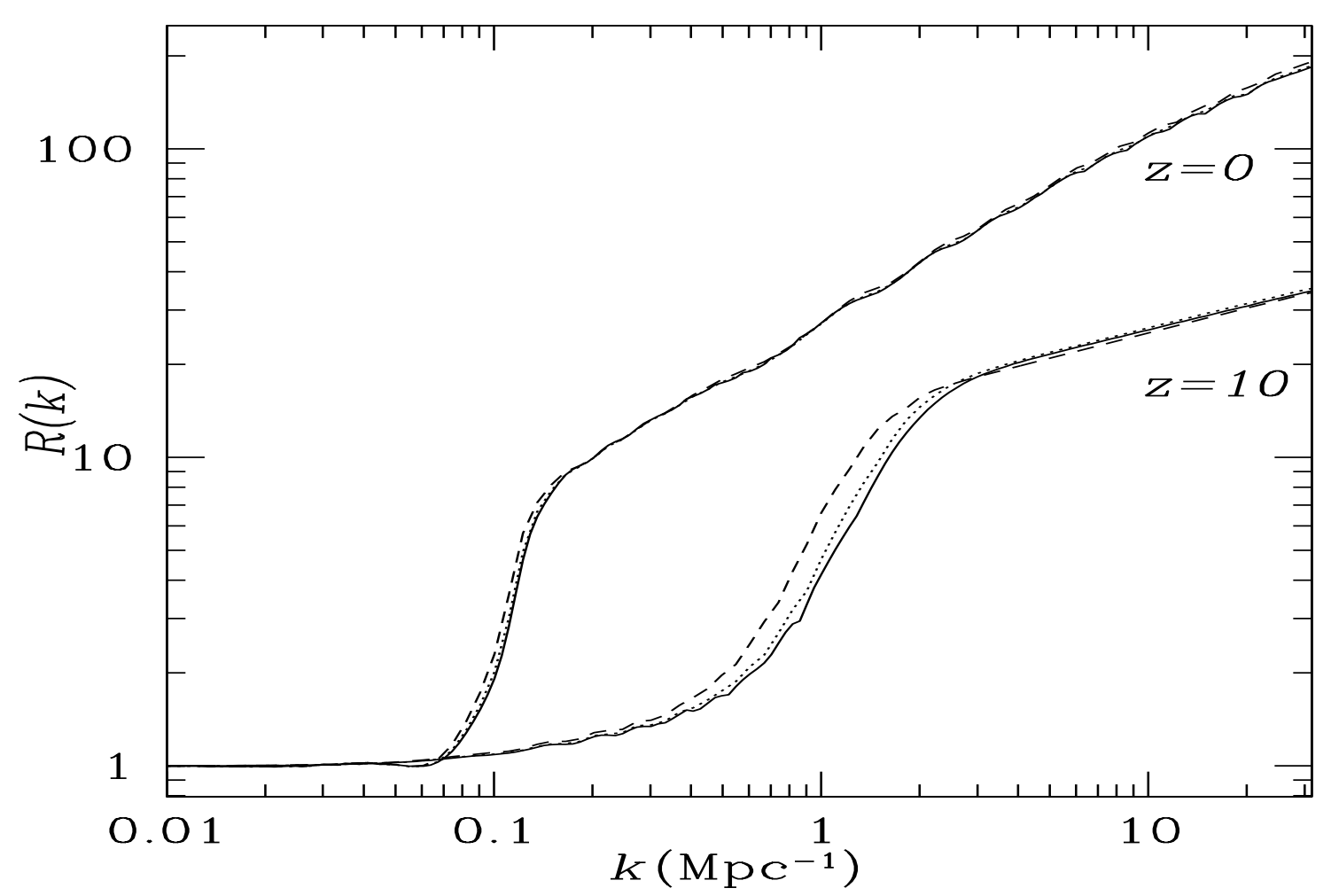

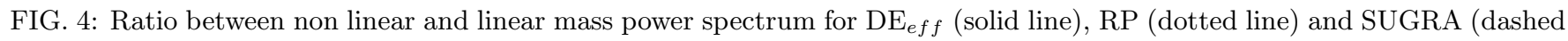
line) with $w_{0}=-0.9$ as function of wavenumber $k$ for two redshift.

\section{THE LINE OF SIGHT DISTRIBUTION OF THE BISPECTRUM SIGNAL}

The integrand in Eq. (18) is built out of a geometrical factor $D$ made of distances and a derivative factor $F$ based on the cosmological fluctuations, this two factors are defined as

$$
D(z)=\frac{r\left(z_{l s s}\right)-r(z)}{r\left(z_{l s s}\right) r^{3}(z)}, F(l, z)=\left[\frac{\partial P_{\Psi}(k, z)}{\partial z}\right]_{k=\frac{l}{r(z)}} .
$$

In this Section we describe and study their relevance for probing the dark energy dynamics.

The factor $D$ is not significantly sensitive to the dark energy redshift dynamics. The reason is that the dependence of $w$ on $z$ gets redshift averaged through the distance integral (44); the latter gets the dominant contribution from the lowest redshifts, say $z \lesssim 0.1$, where all cosmologies converge to the same behavior. Thus, the behavior of $w$ at higher redshifts yields a negligible contribution to the distances, even if the differences are relevant as in Fig. 11 In Fig. 2 we plot $D$ for the three cosmologies defined in Section III showing explicitly the poor sensitivity of $D$ to the $w(z)$ behavior.

The most important contribution in the integral (18) comes from the gravitational potential fluctuation derivative term $F$; we now study its behavior along the line of sight. For a given multipole $l$, and coming from high redshift $z, d P_{\Psi} / d z$ is probed from large to small scales $\lambda(z)=2 \pi r(z) / l$. Let us study the asymptotic regimes $z \rightarrow \infty$ and $z \rightarrow 0$. In the first case, for multipoles of the order $10^{2}$, at very high $z$ the scale $\lambda$ is outside the horizon; in this regime, if the Universe is matter or radiation dominated the potential is constant, so that $F$ vanishes. In the second case, $\lambda \rightarrow 0, F$ vanishes again simply because the power spectrum derivative is probed at infinite wavenumbers. Thus the relevant bispectrum signal must come from some region in between these two asymptotic regimes. This is evident in Fig. 3 where we plot the integrand of $Q(l)$ for different multipoles in the SUGRA scenario described in Section II as a function of the redshift. As $z$ comes down from infinity, the scale $\lambda$ decreases so that eventually it meets the growing comoving scale corresponding to the horizon, $1 / a H$; from that point $\lambda(z)$ becomes a sub-horizon comoving cosmological scale. In this regime, the gravitational potential decreases first, because of the free-streaming of the underlying matter density fluctuations. This feature is visible in Fig. 3 as the tail of the curve at $d P_{\Psi} / d z>0$ coming from high $z$. As $z$ gets smaller, eventually $\lambda(z)$ matches the scale which is entering the non-linear phase at 
the epoch $z$; at this point the derivative changes sign, $d P_{\Psi} / d z<0$, since the power is now increasing with time, i.e. as $z$ decreases. Thus, the negative peaks in Fig. 3 map the non-linear region of the spectrum. As $z$ is reduced to zero, the power vanishes approaching infinite wavenumbers as described above. Thus, the $F$ term yields a non-zero contribution to $Q(l)$ in Eq. (18) only in a redshift interval around the epoch corresponding roughly to the redshift $z_{b s}$ satisfying the relation

$$
\frac{l}{r\left(z_{b s}\right)}=k_{N L}\left(z_{b s}\right),
$$

where the left hand side is purely geometric, while $k_{N L}$ represents the scale entering the non-linear regime at $z_{b s}$.

Now, the effect we just described turns out to be most interesting in at least three aspects. First, as it can be seen in Fig. 3] for multipoles between 1000 and 100 the solution to Eq. (20) is in a redshift interval extremely interesting for the onset of cosmic acceleration, say between 0.1 and 2, respectively. Second, the peak cuts out the present epoch: this means that the bispectrum integral probes the cosmological perturbation dynamics at the relevant redshift for cosmic acceleration, independently on the present. Third, for a fixed multipole $l$, the amplitude of the signal reflects the perturbation dynamics at the epoch $z_{b s}$, which in turn reflects the cosmological expansion rate at that epoch.

In terms of the dark energy properties, the three points above mean that the dark energy equation of state is probed at all the relevant redshifts where it significantly affects the cosmological expansion rate, but independently on its value today; this is a very important feature since it tends to remove the common problem which arises when trying to constrain dark energy models with the CMB, i.e. that the relevant effect is made at the lowest redshifts where all the models converge to the same behavior. We saw an example of such occurrence in the factor $D$ above, where the distances in dark energy cosmologies are essentially dominated by the present dark energy equation of state value $w_{0}$, which greatly reduces the sensitivity to the $w(z)$ behavior at higher redshifts. Indeed, that is the reason why the CMB power spectrum is quite sensible to the effective dark energy equation of state $w_{e f f}$, but poorly on its redshift evolution [33]. In the bispectrum, on the other hand, the signal comes from redshifts strictly greater than 1, cutting out the present, and this is certainly important to discriminate between the different models proposed to explain the evidence for cosmic acceleration. Specifically, if $w(z)$ is different as in the models exposed in Section III we expect to see a difference in the bispectrum signal, greatly enhanced with respect to the one in the ordinary CMB power spectrum, as we show explicitly in the next Section.

Before concluding, we comment on the role of the description we adopt for the non-linear tail of the density

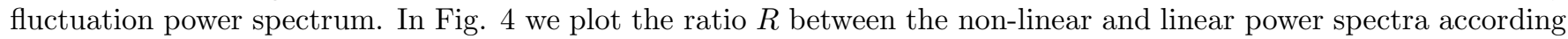
to the recipe adopted here [40], for the dark energy models we consider here. The three models yield a similar behavior in $R$, with some difference in the scale at which $R$ rises for the onset of non-linearity, which in SUGRA occurs at lower wavenumbers for a fixed $z$; as we see in the next Section, that is not induced by the particular recipe adopted for the non-linear mapping of the spectrum, but merely reflects the linear perturbation dynamics, inducing at any given epoch a larger fluctuation amplitude in SUGRA with respect to the RP and $\mathrm{DE}_{\text {eff }}$ cases, slightly shifting the non-linear rise of the spectrum toward smaller multipoles. On the other hand, we will see that the mechanism leading to the sensitivity of the bispectrum to the dark energy equation of state redshift behavior is different, depending substantially only on the time derivative of the linear perturbation spectrum.

\section{SOLVING FOR $w(z)$ WITH THE CMB SPECTRUM AND BISPECTRUM}

In this Section we illustrate the phenomenology described above in the dark energy scenarios defined in Section by computing explicitly the bispectrum signal as a function of the multipole $l$. On small multipoles, i.e. large angles, the corresponding scales are outside the horizon, in linear regime, and $Q(l)>0$. On the other hand, on sufficiently large multipoles, $Q(l)$ probes sub-horizon scales where the non-linear regime dominates, and it has negative sign. The transition is located at the angular scale where the contributions from the scales in linear and non-linear regime balance in the integrand, so that $Q(l)$ is zero. For simplicity, here we evaluate the bispectrum by taking equal multipoles, $l_{1}=l_{2}=l_{3}=l$. This is equivalent to probe the CMB pattern with equilateral triangles of different sizes corresponding roughly to $180 / l$ degrees; that is certainly not the only choice, but it is enough for our purposes here. Eq. (17) simplifies as

$$
B_{l}=3 l(l+1) \sqrt{\frac{(2 l+1)^{3}}{4 \pi}}\left(\begin{array}{lll}
l & l & l \\
0 & 0 & 0
\end{array}\right) C_{l}^{P} Q(l) .
$$

The bispectra in the three dark energy models described in Section $[$ are plotted in Fig. 5 In Fig. 6 we plot the corresponding CMB angular power spectra, together with the binned WMAP data. As it is evident, the models are 
almost degenerate in the CMB spectra, but can be resolved by looking at the bispectrum. Indeed, for the reason we explain in detail below, the angular location at which the linear and non-linear bispectrum contributions balance in the SUGRA model is shifted by several tens of multipoles toward smaller angular scales with respect to the RP and $\mathrm{DE}_{\text {eff }}$ cases, while in the ordinary CMB spectrum the shift is of the order of a few multipoles; this indicates that the bispectrum is almost 10 times more effective than the ordinary CMB angular power spectrum in discriminating the redshift dependence of the dark energy equation of state.

The three models yield equal expansion rate at the present, but as we pointed out in the previous Section, the bispectrum reflects the perturbation dynamics, and thus the cosmological expansion rate, in the interval $0.1 \lesssim z \lesssim 2$, independently on the present, in the multipole range $1000 \gtrsim l \gtrsim 100$. In Fig. [7 we show the perturbation growth factor (13) in the three models, normalized to the same value at present. As it is evident, the curve corresponding to the SUGRA case is sensibly larger at almost all epochs. Correspondingly, the change at low redshift, i.e. when the dark energy starts to dominate the expansion, is stronger in the SUGRA scenario, inducing a larger amplitude for the $d P_{\Psi} / d z>0$ tail in the line of sight integral (18). For the same reason, the bispectrum contribution coming from the gravitational bispectrum time derivative on non-linear scales has lower amplitude, simply because the rise due to the onset of non-linearity has to overcome the gravitational potential decay, which is stronger in the SUGRA with respect to the $\mathrm{RP}$ and $\mathrm{DE}_{\text {eff }}$ cases.

The net effect is that, for a given multipole $l$, the larger is the value of $w$ at the relevant epoch, the larger is the contribution from the linear regime decay of the gravitational potential. Thus, the scale at which the non-linear power balances the linear one is shifted toward larger wavenumber, i.e. larger multipoles, as in Fig. 5. Specifically, the cusp is located at $l=361,362$ for the $\mathrm{DE}_{\text {eff }}$ and RP models, respectively, and at $l=412$ for SUGRA. As we already mentioned, the corresponding shift in the CMB angular power spectrum in Fig 6 is just a few multipoles. Note that even if we increase the present equation of state in the RP case to the border of the current constraints, say at $w_{0}=-0.78$, the cusp moves to $l=390$, still well below the SUGRA case, simply because no RP Quintessence model, within the current constraint, yield a $w(z)$ behavior as the SUGRA one in Fig. 1

We conclude that the effect we pointed out in the previous Section represents a new and relevant feature induced by the dark energy dynamics on the CMB, which is worth taking in serious consideration in future observations: it causes a shift of several tens of multipoles in the CMB bispectrum arising in Quintessence scenarios yielding at the present the same equation of state, the latter being well within the range allowed by the current constraints. The strength of this result is evident when comparing the CMB spectra and bispectra in the different scenarios; the projection shift in the bispectra is about a factor 10 stronger than the corresponding one in the angular shift in the ordinary $\mathrm{CMB}$ spectrum.

\section{SUMMARY AND CONCLUDING REMARKS}

The recent evidence for cosmic acceleration is one of the greatest surprises in modern cosmology, and imposes the introduction of a gravitationally repulsive force acting on cosmological scales, known as dark energy [1, 3]. In the wide set of ideas proposed to explain this occurrence (see [17]), a class of models describes the dark energy as a self-interacting scalar field, the Quintessence, featuring a potential which provides the vacuum energy density required to explain the observed level of cosmic acceleration; several shapes for the Quintessence potential have been proposed out of super-symmetry/gravity breaking models [15, 16]. These scenarios predict characteristic time variations of the dark energy equation of state $w[18$. By getting data on the redshift evolution of $w$ throughout the relevant interval for cosmic acceleration, say $z \lesssim 2$, we can greatly restrict the range of models allowed to explain the dark energy and cosmic acceleration, getting constraints on the fundamental physics behind this process [19].

From this point of view, it is important to focus on the existing cosmological observables and build up new ones which are able to probe the cosmological expansion during the onset of cosmic acceleration. The obvious, cosmological process which occurs at similar redshift is the structure formation, that can be probed statistically by the weak gravitational lensing distortions which makes on background light [21, 38]. The latter effect has been recently observed and represents a promising probe for cosmology [22, 24]. In this work we focus on the weak lensing effect induced by structure formation on the background CMB anisotropies [25, 27]. We look at the bispectrum, built out of the third order moment of CMB anisotropy statistics, because if the latter is Gaussian at last scattering as it appears from recent $\mathrm{CMB}$ observations [26], then the third moment is zero within cosmic variance, with additional power imprinted by the weak lensing of the structures along the line of sight of CMB photons: the latter is expressed as a redshift integral between the last scattering and the present involving cosmological distances and the redshift derivative of the cosmological gravitational potential, induced the structures themselves [25, 28, 29, 39, 42].

We study the redshift behavior of the bispectrum signal arising from the correlation between weak lensing and ISW effect; that is made by two main contributions, deriving from the linear and non-linear regimes of the density 


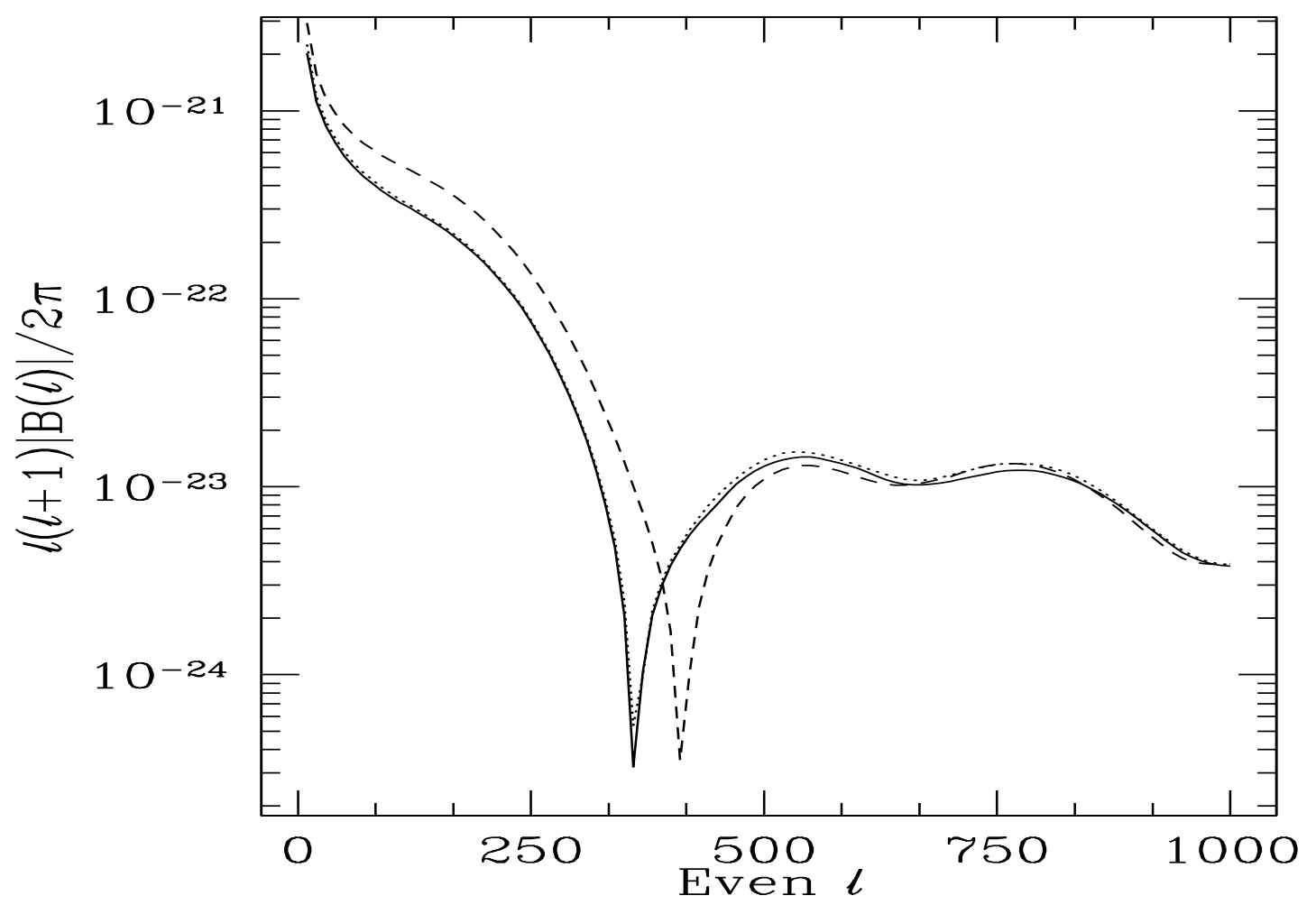

FIG. 5: Absolute value of dimensionless bispectrum for $\mathrm{DE}_{\text {eff }}$ (solid line), RP (dotted line) and SUGRA (dashed line) with $w_{0}=-0.9$ as function of multipole $l$.

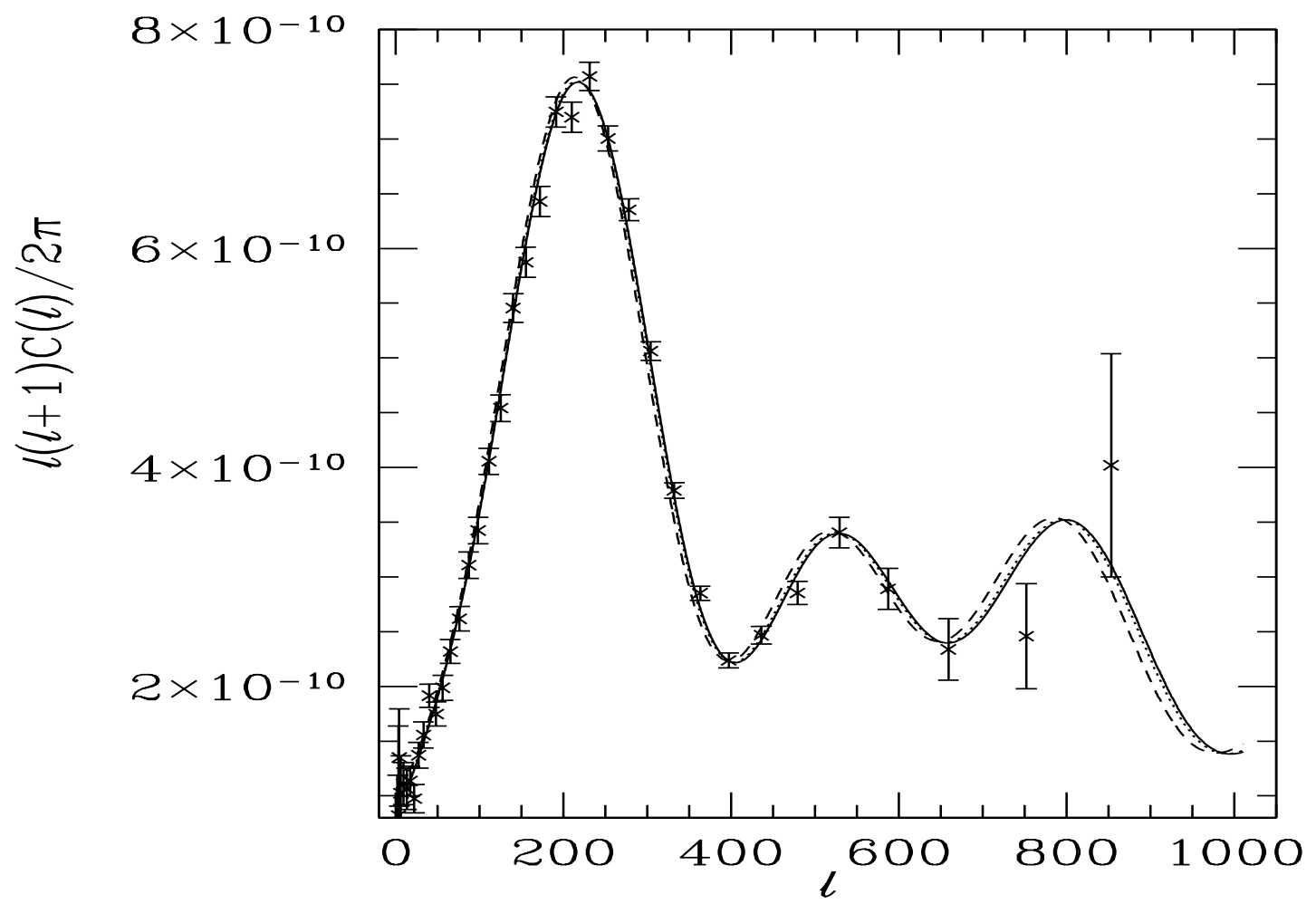

FIG. 6: Dimensionless power spectra for our three models as function of multipole $l$, notice that the degeneration between models is greater than the bispectrum. All the models are normalized to WMAP data. Error bars are the binned WMAP data. 


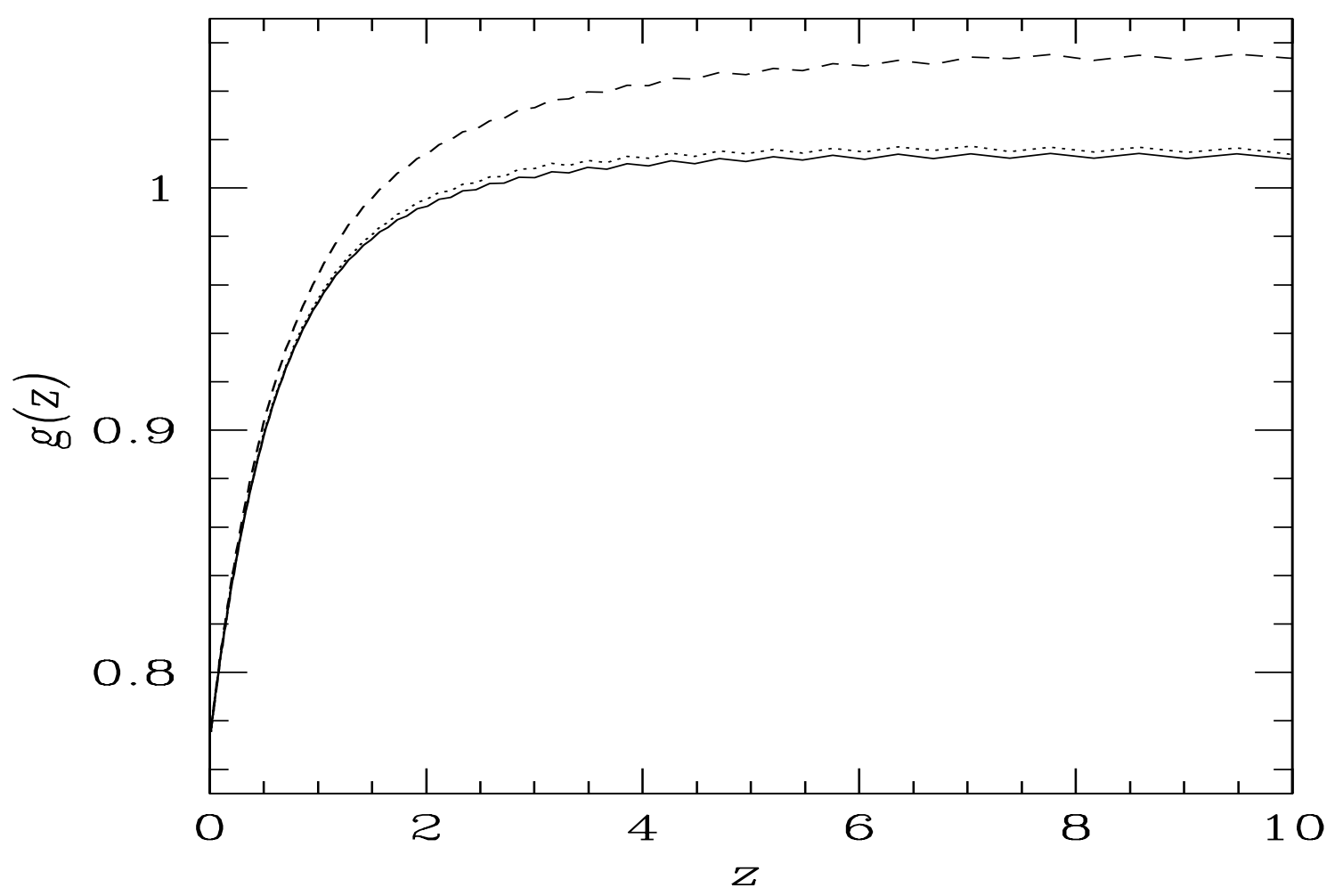

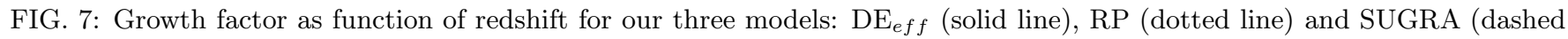
line). We have an appreciable difference between SUGRA and other two models in spite of the same value of $w_{0}$.

perturbations, yielding opposite signs after the horizon crossing: while the linear regime makes the gravitational potential decreasing in time, the non-linear one, which can be described accordingly to the existing semi-analytical recipes 40, 41], yields a growth. Moreover, the integrand gets to zero at high and low redshift, and the relevant contribution, accounting for both the linear and non-linear regimes, comes from a narrow redshift interval centered around the typical epochs of structure formation, coinciding with the onset of cosmic acceleration. As a result, the CMB bispectrum is quite sensitive to the cosmic expansion rate at that redshift, i.e. on the dark energy equation of state at that time, independently on its present value. That represents a new and important aspect of the CMB as a dark energy probe, going in the direction of removing the effect coming from the dark energy evolution at the lowest redshifts, where all the models have to converge in order to reproduce the observed amount of cosmic acceleration 33. To illustrate this effect, we considered two Quintessence scenarios both having present equation of state close to -1 , but markely different in the past, as a result of the different potential shapes: the shallow inverse power law and the steep one including an exponential correction. The latter scenario generally predicts an abrupt change of the dark energy equation of state at $z \lesssim 1$, dropping from values which can be as high as -0.2 , while in the first one the $w$ dynamics yield variations at the $10 \%$ level. As a result, the gravitational potential decay in linear regime gets enhanced in the SUGRA model with respect to the RP one; correspondingly, the scale at which the non-linear part of the spectrum balances the linear one is shifted toward gets smaller, subtending smaller angles in the sky. This induces a projection feature making the bispectra in the two scenarios shifted by several tens of multipoles, i.e. 10 times stronger than the corresponding shift in the ordinary CMB power spectrum.

We did not address here how much this effect is robust against variations in the other cosmological parameters. However, since it is mainly a projection feature, we argue that it could be mimicked by variations in the cosmological parameters inducing geometric features in the CMB anisotropies, such as the overall cosmic geometry, Hubble constant etc. On the other hand, it is important to note that such variations would affect similarly both the angular power spectrum and the bispectrum. We remark that the effect we report here affects the CMB bispectrum much more efficiently than the CMB spectrum, and this is made possible by three key features which are difficult to reproduce by varying the other cosmological parameters: $(i)$ the fact that the bispectrum signal induced by forming structures through weak lensing probes a narrow redshift interval which does not include the present, $(i i)$ the unique prediction of the dark energy cosmology, i.e. that one of the cosmological parameter, i.e. the equation of state, is time varying, and (iii) that different dark energy scenarios generally predict different time variations of the equation of state. For 
this reason we think that it will be useful to take into account the effect we pointed out here when dealing with data provided by the next, high resolution and high sensitivity cosmological probes such as Planck, CMBpol, as well as the weak lensing surveys which will be carried out in the next decade.

\section{ACKNOWLEDGMENTS}

We thank Licia Verde and Eric Linder for important suggestions. Fabio Giovi would like to thanks Pier Stefano Corasaniti for useful discussions, Andrea Ferrara and Ruben Salvaterra for very important comments while this work was in progress. Carlo Baccigalupi is grateful to Martin White for helpful comments.

\section{APPENDIX A: WIGNER 3J SYMBOLS}

The Wigner 3J symbols (W3J) come out from the composition of three spherical harmonics

$$
\begin{aligned}
G_{m_{1} m_{2} m_{3}}^{l_{1} l_{2} l_{3}} & =\int d^{2} \hat{n} Y_{l_{1} m_{1}}(\hat{n}) Y_{l_{2} m_{2}}(\hat{n}) Y_{l_{3} m_{3}}(\hat{n}) \\
& =\sqrt{\frac{\left(2 l_{1}+1\right)\left(2 l_{2}+1\right)\left(2 l_{3}+1\right)}{4 \pi}}\left(\begin{array}{ccc}
l_{1} & l_{2} & l_{3} \\
0 & 0 & 0
\end{array}\right)\left(\begin{array}{ccc}
l_{1} & l_{2} & l_{3} \\
m_{1} & m_{2} & m_{3}
\end{array}\right),
\end{aligned}
$$

Eq. (A1) represent the Gaunt integral. The W3J has a closed formula only for a few combinations of $l_{1}, l_{2}$ and $l_{3}$ and $m_{1}, m_{2}$ and $m_{3}$; for the most general case we need a recursion relation, to be able to compute the whole set of W3J. The recursive relation for W3J is computed for fixed $l_{1}, l_{2}, l_{3}, m_{1}$ and for the other possible values of $m_{2} ; m_{3}$ is given by the relation $m_{3}=-\left(m_{1}+m_{2}\right)$ and if this equality is not verified, the W3J are identically zero. The recursive relation $[43$ ] is

$$
C\left(m_{2}+1\right) g\left(m_{2}+1\right)+D\left(m_{2}\right) g\left(m_{2}\right)+C\left(m_{2}\right) g\left(m_{2}-1\right)=0
$$

where

$$
g\left(m_{2}\right)=\left(\begin{array}{ccc}
l_{1} & l_{2} & l_{3} \\
m_{1} & m_{2} & m_{3}
\end{array}\right)
$$

are the W3J as function of $m_{2}$, and the coefficients $C\left(m_{2}\right)$ and $D\left(m_{2}\right)$ are defined by

$$
\begin{aligned}
& C\left(m_{2}\right)=\sqrt{\left(l_{2}-m_{2}+1\right)\left(l_{2}+m_{2}\right)\left(l_{3}+m_{3}+1\right)\left(l_{3}-m_{3}\right)} \\
& D\left(m_{2}\right)=l_{2}\left(l_{2}+1\right)+l_{3}\left(l_{3}+1\right)-l_{1}\left(l_{1}+1\right)+2 m_{2} m_{3} .
\end{aligned}
$$

The usual choice for the starting values of W3J at the boundaries is $g\left(m_{2 \text { min }}\right)=g\left(m_{2}\right.$ max $)=1$ where $m_{2}$ min and $m_{2 \max }$ are respectively the smaller and the largest values of $m_{2}$, are

$$
\begin{aligned}
& m_{2 \min }=\max \left[-l_{2},-\left(l_{3}+m_{1}\right)\right], \\
& m_{2 \max }=\min \left[l_{2}, l_{3}-m_{1}\right] .
\end{aligned}
$$

It is also possible to work only with the forward recursion, in this way it is sufficient to normalize the whole set of symbols with the normalization condition

$$
\sum_{m_{i}=m_{2} \min }^{m_{2 \max }}\left(2 l_{1}+1\right) g^{2}\left(m_{i}\right)=1
$$

and the phase convention

$$
\operatorname{sign}\left[g\left(m_{2 \max }\right)\right]=(-1)^{l_{2}-l_{3}-m_{1}} .
$$

The W3J have an analytical expression in terms of factorials, when $m_{1}=m_{2}=m_{3}=0$ and $L=\sum_{i=1}^{3} l_{i}$ is even:

$$
\left(\begin{array}{ccc}
l_{1} & l_{2} & l_{3} \\
0 & 0 & 0
\end{array}\right)=(-1)^{L / 2} \frac{(L / 2) !}{\sqrt{(L+1) !}} \prod_{i=1}^{3} \frac{\sqrt{\left(L-2 l_{i}\right) !}}{\left(L / 2-l_{i}\right) !}
$$


For odd $L$ the W3J is zero. Since the computation of factorial may be hard at high multipoles, we can use the Gosper factorial approximation:

$$
n ! \simeq \sqrt{\left(2 n+\frac{1}{3}\right) \pi}\left(\frac{n}{e}\right)^{n} .
$$

in this way we can rewrite Eq. A10 in a computational simpler form

$$
\left(\begin{array}{ccc}
l_{1} & l_{2} & l_{3} \\
0 & 0 & 0
\end{array}\right) \simeq\left(-\frac{L}{L+1}\right)^{L / 2} \frac{1}{(6 L+7)^{1 / 4}}\left(\frac{3 e}{\pi} \frac{3 L+1}{L+1}\right)^{1 / 2} \prod_{i=1}^{3} \frac{\left(6 L-12 l_{i}+1\right)^{1 / 4}}{\left(3 L-6 l_{i}+1\right)^{1 / 2}} .
$$

[1] A. G. Riess et al., Astrophys. J. 116, 1009 (1998), S. Perlmutter et al., Astrophys. J. 517, 565 (1999).

[2] S. Dodelson et al., Astrophys. J. 572, 140 (2002), W. J. Percival et al., Mon. Not. R. Astron. Soc. 337,1068 (2002).

[3] C. L. Bennett et al. 2003, Astrophys. J. in press, preprint astro-ph/0302207 D. N. Spergel and D. M. Goldberg, preprint astro-ph/0302209 (2003).

[4] W. L. Freedman et al., Astrophys. J. 553, 47 (2001).

[5] B. Ratra and P. J. E. Peebles, Phys. Rev. D37, 3406 (1988).

[6] C. Wetterich, Nucl. Phys. B302, 668 (1988).

[7] K. Coble, S. Dodelson and J. A. Frieman, Phys. Rev. D55, 1851 (1997).

[8] P. G. Ferreira and M. Joyce, Phys. Rev. D58, 023503 (1998).

[9] A. R. Liddle and R. J. Scherrer, Phys. Rev. D59, 023509 (1999).

[10] P. J. Steinhardt, L. Wang and I. Zlatev, Phys. Rev. D59, 123504 (1999).

[11] S. M. Carroll, Phys. Rev. Lett. 81, 3067 (1998).

[12] T. Chiba, Phys. Rev. D60, 083508 (1999); J. P. Uzan, Phys. Rev. D59, 123510 (1999); F. Perrotta, C. Baccigalupi and S. Matarrese, Phys. Rev. D61, 023507 (2000); N. Bartolo and M. Pietroni, Phys. Rev. D61, 023518 (2000); C. Baccigalupi, S. Matarrese and F. Perrotta, Phys. Rev. D62, 123510 (2000); V. Faraoni, Phys. Rev. D62, 023504 (2000); G. Esposito-Farese and D. Polarski, Phys. Rev. D63, 063504 (2001); A. Riazuelo and J. P. Uzan, Phys. Rev. D66, 023525 (2002); F. Perrotta and C. Baccigalupi, Phys. Rev. D59 123508 (2002).

[13] L. Amendola, Phys. Rev. D62, 043511 (2000); L. Amendola, C. Quercellini, D. Tocchini-Valentini and A. Pasqui, Astrophys. J. Lett. 583 L53 (2003); S. Matarrese, M. Pietroni and C. Schimd, preprint astro-ph/0305224 (2003).

[14] C. Armendariz-Picon, V. Mukhanov and P. J. Steinhardt, Phys. Rev. D63, 103510 (2001); R. R. Caldwell, Phys. Lett. B545, 23 (2002); M. Malquarti, E. J. Copeland and A. R. Liddle, Phys. Rev. D68, 023512 (2003).

[15] A. Masiero, M. Pietroni and F. Rosati, Phys. Rev. D61, 023504 (2000).

[16] P. Brax and J. Martin, Phys. Rev. D61, 103502 (2000).

[17] T. Padmanabhan, Phys.Rep. 380, 235 (2003); P. J. E. Peebles and B. Ratra, Rev. Mod. Phys. 75,599 (2003).

[18] P. S. Corasaniti and E. J. Copeland, Phys. Rev. D67, 063521 (2003).

[19] E.V. Linder, Phys. Rev. Lett. 90, 091301 (2003).

[20] D. N. Spergel et al. Astrophys. J. in press, preprint astro-ph/0302209 (2003).

[21] M. Bartelmann and P. Schneider, Phys. Rep. 340, 291 (2001).

[22] L. van Waerbecke et al., Astron. Astrophys. 358, 30 (2000); D. Bacon, A. Refregier and R. Ellis Mon. Not. R. Astron. Soc. 318625 (2000); G. Wilson, Kaiser N. and G. A. Luppino, Astrophys. J. 556, 601 (2001); D. M. Wittmann, J. A. Tyson, D. Kirkman, I. Dell'Antonio and G. Bernstein, Nature 405, 143 (2000); R. Maoli et al., Astron. Astrophys. 368766 (2001); L. van Waerbecke et al., Astron. Astrophys. 374, 757 (2001).

[23] M. Bartelmann, M. Meneghetti, F. Perrotta, C. Baccigalupi and L. Moscardini, Astron. Astrophys. in press, preprint astro-ph/0210066 (2003); N. N. Weinberg and M. Kamionkowski, Mont. Not. R. Astron. Soc. 341, 251 (2003); F. Bernardeau, Rep. Progr. Phys. 66, 691 (2003); M. Bartelmann, F. Perrotta and C. Baccigalupi, Astron. Astrophys. 396, 21 (2002); W. Hu, Phys. Rev. D 66, 083515 (2002); D. Huterer, Phys. Rev. D 65, 063001 (2002).

[24] A. Refregier et al., Ann. Rev. Astron. Astrophys in press, preprint astro-ph/0304419 (2003).

[25] W. Hu, Phys. Rev. D 62, 043007 (2000).

[26] E. Komatsu et al., Astrophys. J. in press, preprint astro-ph/0302223 (2003).

[27] W. Hu and M. White, Phys. Rev. D56, 596 (1997).

[28] E. Komatsu and D. N. Spergel, Phys. Rev. D63, 063002 (2001).

[29] L. Verde and D. N. Spergel, Phys. Rev. D 65, 043007 (2002).

[30] J. Weller and A. M. Lewis, preprint astro-ph/0307104 (2003).

[31] F. Perrotta and C. Baccigalupi, Phys. Rev. D59 123508 (1999).

[32] U. Seljak and M. Zaldarriaga, Astrophys.J. 469, 437 (1996).

[33] R.R. Caldwell, M. Doran, C.M. Muller, G. Schafer and C. Wetterich, Astrophys. J. Lett. 591, L75 (2003); C. Baccigalupi, A. Balbi, S. Matarrese, F. Perrotta and N. Vittorio, Phys. Rev. D65, 063520 (2002); P. S. Corasaniti and E. J. Copeland, Phys. Rev. D65, 043004 (2002); M. Doran, M. Lilley, J. Schwindt and C. Wetterich, Astrophys. J. 559, 501 (2001). 
[34] A. Balbi, C. Baccigalupi, F. Perrotta, S. Matarrese and N. Vittorio, Astrophys. J. Lett. 588, L5 (2003).

[35] M. Rees and D. W. Sciama, Nature 217, 511 (1968).

[36] Ya. B. Zel'dovich and R. A. Sunyaev, Astrophys. Space Sci. 4, 301 (1969).

[37] J. P. Ostriker and E. T. Vishniac, Astrophys. J. Lett. Ed. 306, L51 (1986).

[38] N. Kaiser, Astrophys. J. 388, 272 (1992).

[39] D. N. Spergel and D. M. Goldberg, Phys. Rev. D59, 103001 (1999); D. M. Goldberg and D. N. Spergel, Phys. Rev. D59, 103002 (1999).

[40] C. P. Ma, Astrophys. J. 508, L5 (1998); C. P. Ma, R. R. Caldwell, P. Bode and L. Wang, Astrophys. J. Lett. 621, L1 (1999).

[41] R. E. Smith et al., Mon. Not. R. Astron. Soc. 341, 1311 (2003).

[42] A. Cooray, W. Hu, Astrophys. J. 534, 533 (2000).

[43] K. Schulten and R. G. Gordon, J. Math. Phys. 16, 196 (1975). 